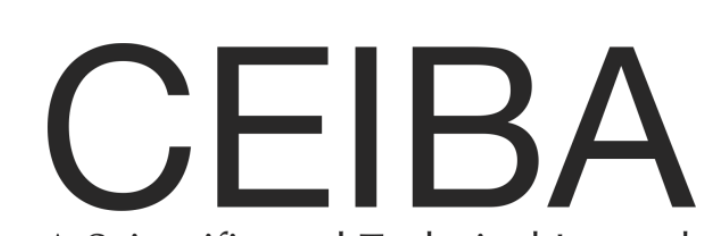

A Scientific and Technical Journal

Published by Zamorano

\title{
0843
}

\section{Producción de tilapia (Oreochromis niloticus L.) en la etapa de engorde con dos estrategias de alimentación}

\author{
Patricio E. Paz \\ Escuela Agrícola Panamericana \\ P.O. Box 93, Tegucigalpa, Honduras \\ Alejandra Danelia Martinez Turcios \\ Escuela Agrícola Panamericana \\ P.O. Box 93, Tegucigalpa, Honduras \\ José Ignacio Chávez Chávez \\ Escuela Agrícola Panamericana \\ P.O. Box 93, Tegucigalpa, Honduras
}

Fecha de publicación: Enero 30, 2019 


\section{Patricio E. Paz, Alejandra Danelia Martinez Turcios \& José Ignacio Chávez Chávez \\ Producción de tilapia (Oreochromis niloticus L.) en la etapa de engorde con dos estrategias de alimentación \\ Ceiba 0843: 1-6}

DOI 10.5377/ceiba.v0i0843.5824

\section{Publicado en el 2019 por}

Escuela Agrícola Panamericana, Zamorano

P.O. Box 93

Km 30 Tegucigalpa a Danlí,

San Antonio de Oriente, Francisco Morazán, Honduras

Ceiba es la revista científica y tecnológica de la Escuela Agrícola Panamericana, Zamorano, publicada desde 1950. A partir del 2019, Ceiba se publica exclusivamente de manera electrónica y en el formato de publicación continua; esto con el propósito de acortar el tiempo entre aceptación de un artículo y su publicación y que los autores puedan diseminar rápidamente los resultados de su investigación. Aceptamos artículos en español o en inglés relacionados con todas las áreas de la agricultura: agricultura tropical, recursos naturales, botánica, biología, ecología, desarrollo rural, forestales, economía agrícola, agronegocios, descripciones de nuevas especies, agroindustria, protección vegetal, notas científicas (plagas nuevas, observaciones interesantes) y resúmenes de tesis de los estudiantes de la Escuela Agrícola Panamericana. Ocasionalmente se publicarán revisiones bibliográficas y resúmenes de conferencias.

Los artículos para ser considerados para publicación tienen que ser originales y no deben haber sido publicados ni sometidos a otras revistas. Una vez sometidos a Ceiba tampoco deben someterse a otras revistas. Los autores deben indicar en su primer envío del artículo que cumplen con esta norma. Los artículos serán arbitrados por dos personas antes de aceptarlos para publicación usando el sistema de doble ciego. Ceiba usa revisores externos, el autor puede sugerir los revisores, pero no se garantiza que se usen. Después de ser revisado, los comentarios de los revisores se regresan al autor con el cual se trabaja en el manuscrito si este fue aceptado. Si el artículo es aceptado, se deben hacer las correcciones sugeridas por los revisores y regresarlo al editor en no más de dos semanas.

Los autores deben enviar sus artículos a través del sitio web único de la revista: https://www.lamjol.info/index.php/CEIBA para lo cual deben crear una cuenta en el sitio. Ceiba no dispone de ningún otro sitio para la recepción de manuscritos. En caso de algún problema, los autores pueden enviar por correo electrónico todos los archivos que forman el artículo (cuadros, fotografías, dibujos y gráficas) al editor, Dr. Jesús Orozco, al correo electrónico jorozco@zamorano.edu. Adicionalmente, pueden comunicarse con el editor al teléfono +504 2287-2000 ext 2340.

Ceiba está indexada en Web of Science, latindex y Central American Journals Online (CAMJOL).

Editor: Jesús Orozco

Diagramación: Jesús Orozco y Dafna Díaz

Comité editorial: Ronald D. Cave, Mike D. Owen, José Manuel Mora, Carlos Hernández DíazAmbrona y Abelino Pitty

ISSN 2225-6687

Copyright. Este es un artículo de acceso abierto distribuido bajo los términos de Creative Commons, licencia de atribución no comercial, el cual permite el uso, distribución y reproducción no comercial en cualquier medio sin restricciones, siempre y cuando el autor original y la fuente sean acreditados. https://creativecommons.org/licenses/by-nc/3.0/deed.es. El copyright pertenece a los autores. 


\title{
Producción de tilapia (Oreochromis niloticus L.) en la etapa de engorde con dos estrategias de alimentación
}

\author{
Patricio E. Paz \\ ppaz@zamorano.edu \\ Escuela Agrícola Panamericana \\ P.O. Box 93, Tegucigalpa, Honduras
}

\author{
Alejandra Danelia Martinez Turcios \\ alejandra.martinez@est.zamorano.edu \\ Escuela Agrícola Panamericana \\ P.O. Box 93, Tegucigalpa, Honduras
}

\section{José Ignacio Chávez Chávez \\ joseignaciochavezch94@gmail.com \\ Escuela Agrícola Panamericana \\ P.O. Box 93, Tegucigalpa, Honduras}

\begin{abstract}
Resumen. La tilapia gris (Oreochromis niloticus L.) es una excelente opción para la producción acuícola debido a su resistencia a condiciones adversas, enfermedades y sus hábitos alimenticios. Se evaluaron dos estrategias de alimentación durante la etapa de engorde, la cual finaliza al alcanzar un peso ideal de 1,085 g a la cosecha. Las estrategias de alimentación fueron ad libitum y alimentación al 90 \% del ad libitum. El ensayo se realizó en AquaFinca Saint Peter Fish, Cortés, Honduras. Las variables medidas fueron: índice de conversión alimenticia (ICA), índice de alimentación (IA), días a cosecha (Decos) y ganancia de peso diario (GDP). Las unidades experimentales fueron doce jaulas flotantes, seis jaulas ad libitum y seis jaulas con el $90 \%$ del ad libitum; cada jaula tenía un volumen de 1,380 $\mathrm{m}^{3}$ con una capacidad de 50,000 peces. El análisis estadístico se realizó por medio de una prueba $t(\mathrm{P} \leq 0.05)$. La alimentación ad libitum y al $90 \%$ del ad libitum no presentaron diferencias en ICA (1.88 y 1.82 respectivamente), tampoco se presentaron diferencias en GDP con $6.39 \mathrm{~g}$ ad libitum y $6.16 \mathrm{~g}$ con $90 \%$ del ad libitum. El tratamiento ad libitum presentó un IA de 3.19 y el $90 \%$ del ad libitum de 3.06; Decos fueron 157 y 165 respectivamente. El tratamiento al $90 \%$ del ad libitum, además de representar un ahorro en alimento no tuvo efecto sobre el ICA, pero si se retrasó ocho días para llegar al peso ideal de cosecha con una diferencia de $26,270 \mathrm{~kg}$ de alimento entre ambos tratamientos.
\end{abstract}

Palabras clave: Conversión alimenticia, índice de alimentación.

\section{Production of tilapia (Oreochromis niloticus L.) during grow-out with two feeding strategies}

\begin{abstract}
The gray tilapia (Oreochromis niloticus L.) is an excellent choice for aquaculture due to its resistance to adverse conditions and diseases, and their eating habits. Two feeding strategies were evaluated during the grow-out stage, which ended when the ideal weight of 1,085 g was reached. The feeding strategies were ad libitum and feed at $90 \%$ of ad libitum. The trial was performed at AquaFinca Saint Peter Fish, Cortés, Honduras. The analyzed variables were feed conversation ratio (FCR), specific feeding rate (SFR), days to harvest (Dhar) and daily weight gain (DWG). Twelve floating cages were used, six cages for each feeding strategy. Each cage had a volume of $1,380 \mathrm{~m}^{3}$ and capacity for 50,000 fish. Statistical analysis were performed using Student's $t$ test $(\mathrm{P} \leq 0.05)$. The ad libitum and $90 \%$ of $a d$ libitum did not show differences in FCR (1.88 and 1.82 respectively); no differences in DWG with $6.39 \mathrm{~g}$ in ad libitum and $6.16 \mathrm{~g}$ in $90 \%$ of ad libitum were observed. In SFR ad libitum had 3.19 and 3.06 for $90 \%$ ad libitum, and Dhar was 157 in ad libitum and 165 in $90 \%$ of ad libitum. The $90 \%$ of ad libitum presented lower feed consumption without affecting the FCR and took eight days more to reach the ideal harvest weight with a difference of $26,270 \mathrm{~kg}$ of feed use between both treatments.
\end{abstract}

Key words: Food conversion, specific feeding rate.

\section{Introducción}

La acuicultura es el rubro alimenticio con mayor crecimiento a nivel mundial, generando el $50 \%$ del DOI 10.5377/ceiba.v0i0843.5824 
Paz et al. Ceiba 2019, 0843: 1-6. Producción de tilapia en la etapa de engorde con dos estrategias de alimentación

pescado consumido en el mundo (FAO 2017). Este crecimiento ha sido explicado por las tendencias nutricionales recientes que se adaptan a las propiedades nutricionales de la carne de pescado baja en grasas, abundante en proteína de alta calidad, rica en fósforo, calcio y vitaminas (Luchini 2010) y por la creación de nuevas políticas pesqueras y el incremento de la población (FAO 2017).

En el sector acuícola la tilapia es el sexto producto con mayor importancia económica a nivel mundial, siendo China el mayor productor (El-Sayed 2006). En Centro América, la tilapia es considerado el rubro acuícola de exportación de producto fresco más importante (Meyer y Meyer 2007). En Honduras en particular, la tilapia generó un ingreso por exportación de más de 64 millones de dólares en el 2015 (BCH 2016).

La tilapia gris (Oreochromis niloticus L.), es un pez teleósteo oriundo de África e introducido a regiones tropicales, sub tropicales y ambientes controlados de todo el mundo (Cantor Atlatenco 2007). La tilapia gris al igual que la mayoría de cíclidos se caracteriza por su adaptabilidad al cautiverio y por su tolerancia a condiciones físicas y químicas adversas, bajas concentraciones de oxígeno disuelto y enfermedades (Saavedra 2006). Características nutricionales favorables como su rápido crecimiento y una dieta extensa han hecho que la tilapia gris tenga un lugar importante dentro de la acuicultura (Meyer y Meyer 2007).

Los costos en alimentación durante el engorde de tilapia gris representan el 50-60 \% de los costos totales (Nicovita 2012). Debido a los altos costos en alimentación, es fundamental una evaluación continua de las estrategias de alimentación en la fase de engorde y asegurar que el alimento sea consumido por el pez, de lo contrario, el alimento simplemente se convierte en un fertilizante caro, disminuyendo la rentabilidad del negocio y afectando la calidad del agua (Nicovita 2012).

La estrategia de alimentación ad libitum es popular en nutrición animal ya que minimiza la mano de obra en el reparto de alimento, consigue bajos índices de conversión alimenticia (ICA), elimina los problemas de jerarquía o competencia entre individuos y logra uniformidad de tallas y peso en la población (Caravaca et al. 2003). El concepto de alimentación ad libitum en organismos acuáticos es distinto que en otras especies terrestres ya que los alimentos balanceados pierden sus propiedades al contacto con el agua y por esta razón las raciones son divididas en periodos de tiempo.

La estrategia de la alimentación del $90 \%$ del ad libitum sugiere cubrir en un $90 \%$ la saciedad de los peces; se sabe que el pez consume más alimento del que necesita por lo tanto existe un porcentaje de energía que no es aprovechado de una manera eficiente, con esta estrategia se intenta llegar al punto de máxima eficiencia en cuanto a ICA (Lovell 1989). La teoría de alimentar al $90 \%$ del ad libitum (Lovell 1989) es utilizada y recomendada por el Consejo de Exportación de Soya de los Estados Unidos (USSEC, por sus siglas en inglés) a empresas de todo el mundo.

El objetivo de este estudio fue evaluar dos estrategias de alimentación en etapa de engorde: ad libitum y $90 \%$ del ad libitum en el crecimiento, días a cosecha y la eficiencia en el índice de conversión alimenticia.

\section{Métodos}

El ensayo se llevó a cabo en AquaFinca Saint Peter Fish, en el centro de producción El Cajón, departamento de Cortés, Honduras de diciembre del 2016 a abril del 2017. Esta zona se encuentra a 260 msnm y presenta una precipitación anual de $975-1,200 \mathrm{~mm}$, con temperaturas promedio de $28-30{ }^{\circ} \mathrm{C}$. El Cajón cuenta con espejo de agua de $94 \mathrm{~km}^{2}$ en su nivel máximo. La época con mayor precipitación se presenta en los meses de mayo a octubre y la época de sequía se presenta de diciembre hasta abril.

Los peces utilizados fueron obtenidos de Regal Springs México y trasladados a Honduras. El ensayo se inició sembrando 49,465 peces por jaula (593,578 peces en total) con un peso promedio de $73 \mathrm{~g}$ y un total de biomasa por jaula promedio de $3,609 \mathrm{~kg}$. El estudio fue realizado en seis jaulas flotantes de $18 \mathrm{~m}$ de diámetro con una profundidad de seis metros. Cada jaula tenía capacidad para $1,380 \mathrm{~m}^{3}$ de agua y 50,000 peces a una densidad de 36 peces por metro cúbico. Las jaulas flotantes fueron estructuras circulares de polietileno de 
Paz et al. Ceiba 2019, 0843: 1-6. Producción de tilapia en la etapa de engorde con dos estrategias de alimentación

alta densidad de $25.4 \mathrm{~cm}$, sujetas por un cable de acero. Cada jaula tenía cuatro tipos de malla: externa, interna, ruedo de malla sombra y malla antipájaros. Las mallas antipájaros y los ruedos de malla sombra son estándar, con luz de malla de $7.6 \mathrm{~cm}$ y al $50 \%$, respectivamente. Las unidades experimentales iniciaron con una malla luz externa de $5.7 \mathrm{~cm}$ y una malla interna de $3.5 \mathrm{~cm}$, un mes después las mallas externas e internas fueron cambiadas a una sola malla luz de $5.1 \mathrm{~cm}$. Cada jaula constó de cuatro aireadores tipo aspiración propulsión, encendidos manualmente cuando el oxígeno disuelto era inferior a 3 ppm.

Se suministró alimento balanceado extrusado (Cuadro 1). La alimentación se inició con alimento con $36 \%$ de proteína cruda (PC) y un tamaño de partícula de $4 \mathrm{~mm}$. A los 22 días de ensayo se cambió el alimento a $32 \%$ de PC y un tamaño de $7 \mathrm{~mm}$. A los 83 días de ensayo se finalizó con un $32 \%$ de PC y un tamaño de $9 \mathrm{~mm}$. El índice de alimentación (IA) en ambos tratamientos se inició con $4.9 \%$.

Cuadro 1. Composición del alimento para el último periodo de engorde (datos del fabricante, AquaFeed).

\begin{tabular}{lc} 
Cuadro 1. Composición del alimento para el último periodo de engorde (datos del fabricante, AquaFeed). \\
\hline \multicolumn{1}{c}{ Componentes } & Cantidad en el alimento \\
\hline Proteína & $32 \%$ \\
Humedad & $12 \%$ \\
Ceniza & $10 \%$ \\
Grasa & $6 \%$ \\
Fibra & $5 \%$ \\
Calcio & $1-2 \%$ \\
Fósforo & $0.6 \%$ \\
Energía metabolizable & $3100 \mathrm{kcal} / \mathrm{kg}$ \\
\hline
\end{tabular}

En ambos tratamientos se alimentó durante 15 minutos seis veces al día con periodos de espera de 35 minutos. En la alimentación al $90 \%$ del ad libitum se dividió por ciclos de 10 días para ajustar la cantidad de alimento. El primer día de cada ciclo se alimentó ad libitum, cuantificando la cantidad al final del día y proveyendo el $90 \%$ de esta ración por los siguientes nueve días del ciclo. Este procedimiento se realizó hasta cosecha. El procedimiento de muestreo constó en pesar 10-15 peces por jaula 20 veces durante los 138 días del experimento. Los peces tuvieron un peso de 900-1,020 g, obteniendo un peso promedio por canasta de $12 \mathrm{~kg}$ (Cuadro 2). Con los datos obtenidos en los muestreos, se utilizó la Fórmula 1 para obtener el peso promedio de los peces (g), este peso obtenido a los 138 días. Los peces se cosecharon con un peso promedio de 1,085 g. Al peso promedio de peces en el muestreo se le restó un $3 \%$ debido al margen de error causado por el movimiento de los peces.

Cuadro 2. Formato de muestreo para peces de 900-1,999 g realizado en el ensayo.

\begin{tabular}{cccc}
\hline Repetición & Peso de peces $(\mathbf{k g})$ & № peces & Peso promedio \\
\hline 1 & 11.7 & 12 & 946 \\
2 & 12.4 & 13 & 925 \\
3 & 11.8 & 12 & 954 \\
$\sum$ & 35.9 & 37 & \\
$\bar{X}$ & & & 942 \\
\hline
\end{tabular}

$$
\text { Peso promedio de peces }=\frac{\Sigma \text { peso de peces }(g)}{\Sigma \text { № peces }}-\left[\frac{\Sigma \text { peso de peces }(g)}{\Sigma \text { № peces }} \times 0.03\right]
$$


Paz et al. Ceiba 2019, 0843: 1-6. Producción de tilapia en la etapa de engorde con dos estrategias de alimentación

El índice de conversión alimenticia (ICA), la cantidad de alimento representada en una unidad de peso de alimento que se convierte en peso vivo, se evaluó con la Fórmula 2.

$$
\mathrm{ICA}=\frac{\text { Alimento acumulado por jaula }(\mathrm{kg})}{\text { Biomasa promedio por jaula }(\mathrm{kg})}
$$

Los niveles de oxígeno disuelto a lo largo del estudio se mantuvieron superiores al límite inferior de 2.0 ppt (García Ortega y Calvario Martínez 2008). El oxígeno disuelto fue medido mediante un oxigenómetro Oxyguard; cuando este fue inferior a $3 \mathrm{~g} / \mathrm{L}$ se encendió el sistema de aireación. La temperatura del agua se mantuvo dentro de los parámetros adecuado de producción sugeridos de $24-32{ }^{\circ} \mathrm{C}$ (El-Sayed 2008). Los sólidos totales $(\mathrm{mg} / \mathrm{L})$ fueron medidos mediante gravimetría, este presentó en su mayoría resultados inferiores al límite de $25 \mathrm{mg} / \mathrm{L}$, considerado aceptable (López et al. 2005). La transparencia se midió con un disco Secchi, manteniéndose entre 2.3-3.34 m. El pH se mantuvo entre 6.5-9.

Se utilizó una prueba $t$ en el programa Statistical Analysis System (SAS® 2015) v. 9.4 para evaluar diferencias en el índice de conversión alimenticia (ICA), índice de alimentación (IA), días a cosecha (Decos) y ganancia diaria de peso (GDP).

\section{Resultados}

Los parámetros usados en este estudio indican que es factible adoptar una estrategia de alimentación al $90 \%$ del ad libitum. La estrategia de alimentación al $90 \%$ del ad libitum fue menos eficiente en cuanto a parámetros productivos como índice de alimentación e índice de conversión alimenticia. La estrategia de alimentación al $90 \%$ del ad libitum obtuvo un índice de conversión alimenticia igual a la estrategia de alimentación ad libitum, pero un índice de alimentación más bajo por lo tanto se reducen costos en alimentación, pero se aumentan los días a cosecha hasta en ocho días.

\section{Índice de conversión alimenticia (ICA)}

En la estrategia de alimentación ad libitum el ICA fue de 1.88 y en la estrategia de alimentación al $90 \%$ del ad libitum fue de 1.82. No hubo diferencia entre los tratamientos $(\mathrm{P}>0.05)$. Clark et al. (1990), quienes midieron los efectos de la tasa de alimentación de cinco tratamientos con distintos porcentajes de alimentación, $50 \%, 70 \%, 90 \%$ del ad libitum y $110 \%$ en la etapa de juveniles o preengorde, tampoco encontraron diferencias con una alimentación del 90 \% y $110 \%$ del ad libitum.

\section{Índice de alimentación (IA)}

En la estrategia de alimentación ad libitum el IA fue de 3.19 y en la estrategia de alimentación al $90 \%$ del ad libitum fue de 3.06 indicando una diferencia entre ambas estrategias de alimentación $(\mathrm{P} \leq 0.05)$. El índice de alimentación cambia según la etapa en la que se encuentre el pez y la calidad del alimento (Hepher 1993). Alimentos con mayor porcentaje de proteína presentarán un IA más bajo que alimentos pobres en proteína.

Explotaciones tradicionales, no intensivas, de tilapia utilizan en la etapa de engorde alimentos 20-28\% de proteína alcanzando un IA de $1.1 \mathrm{a} 683 \mathrm{~g}$ de peso vivo en un periodo de seis meses (Cantor Atlatenco 2007). Este estudio, bajo un sistema intensivo de producción, obtuvo un IA promedio entre los dos tratamientos de 3.13 a un periodo de cosecha de seis meses con peso a cosecha de $1,085 \mathrm{~g}$.

\section{Ganancia diaria de peso (GDP)}

Se encontraron diferencias entre los tratamientos $(\mathrm{P} \leq 0.05)$, con ad libitum alcanzando $6.19 \mathrm{~g}$ y $90 \%$ del ad libitum 6.39 g. Brown et al. (2001) no encontraron diferencias entre ganancia diaria de peso entre libitum vs $67 \%$ del ad libitum. Según Hepher (1993), estos resultados confirman que se cumplen los requerimientos nutricionales para mantenimiento y crecimiento del pez con la dieta de $90 \%$ del ad libitum y a su vez presentaron mayor eficiencia que la estrategia de alimentación ad libitum. 


\section{Días a cosecha (Decos)}

Con la estrategia de alimentación ad libitum se cosechó a los 157 días mientras que con la estrategia de alimentación al $90 \%$ del ad libitum se cosechó a los 165 días, resultando en una diferencia de 8 días (P $\leq 0.05)$ hasta alcanzar el peso ideal de cosecha.

\section{Literatura Citada}

BCH (Banco Central de Honduras). 2016. Comercio general de Tilapia. Tegucigalpa, Honduras: Secretaría de Agricultura y Ganadería. Programa Nacional de Desarrollo Agroalimentario. [consultado 2017 may 27]. Disponible en: http://www.bch.hn/download/honduras_en_cifras/hencifras2014_2016.pdf.

Brown CI, Bolivar RB, Jímenez EBT, Szyper JP. 2001. Reduction of feed rations below satiation levels in tilapia pond production. In: McElwee K, Lewis K, Nidiffer M, Buitrago P, editors. Nineteenth Annual Technical Report. Corvallis, Oregon (USA): Pond Dynamics/Aquaculture CRSP. p.21-23.

Cantor Atlatenco F. 2007. Manual de producción de tilapia. Primera edición. Puebla (México): Secretaría de Desarrollo Rural del Estado de Puebla. 135p.

Caravaca Rodríguez FP, Castel Genís JM, Guzmán Guerrero JL, Delgado Pertíñez M, Mena Guerrero Y, Alcalde Aldea MJ, González Redondo P. 2003. Bases de la producción animal. Primera edición. Sevilla, España: Servicios de Publicaciones Universidad de Córdoba. 512p.

Clark JH, Watanabe WO, Ernst DH, Wicklund RI, Olla BL. 1990. Effect of feeding rate on growth and feed conversion of Florida Red tilapia reared in floating marine cages. Journal of the World Aquaculture Society. 21(1):16-24.

El-Sayed AFM. 2006. Tilapia Culture. First edition. Oxfordshire (UK): CABI Publishing. 277p.

FAO (Food and Agriculture Organization of the United Nations). 2017. Acuicultura: Desarrollo de la acuicultura [internet]. Roma: FAO; [consultado 2017 sep 22]. Disponible en: http://www.fao.org/aquaculture/es/.

García Ortega A, Calvario Martínez O. 2008. Manual de buenas prácticas de producción acuícola de tilapia para la inocuidad alimentaria. Primera edición. Mazatlán, México: Centro de Investigación y Alimentación y Desarrollo SENASIC/SAGARPA. 159p.

Hepher B. 1993. Nutrición de peces comerciales en estanques. Tercera edición. México DF (México): Grupo Noriega Editores. 406p.

López Y, Trujillo E, Fonseca MG, Martínez V. 2005. Identificación de las características físicas, químicas y microbiológicas del agua, que se alteran por uso acuícola. México: Centro Interamericano de Recursos del Agua, Facultad de Ingeniería. Universidad Autónoma del Estado de México.

Lovell T. 1989. Nutrition and feeding of fish. Second edition. New York (USA): Springer US. 260p.

Luchini L. 2010. Beneficios nutricionales y de salud del producto "pescado" [internet]. Buenos Aires, Argentina: Dirección de Acuicultura; [consultado 2017 jul 15]. Disponible en: http://www.agroindustria.gob.ar/sitio/areas/acuicultura/publicaciones/_archivos/000000_Desarrollo s Acu \%C3 \%ADcolas/101210 Beneficios nutricionales y de salud del producto pescado.pdf

Meyer D, Meyer ST. 2007. Reproducción y cría de alevines de tilapia: Manual Práctico. Honduras: Zamorano. 48p. 
Paz et al. Ceiba 2019, 0843: 1-6. Producción de tilapia en la etapa de engorde con dos estrategias de alimentación

Nicovita. 2012. Manual de Crianza de Tilapia. Lima, Perú. [consultado 2019 ene 11]. Disponible en: http://www.industriaacuicola.com/biblioteca/Tilapia/Manual de crianza de tilapia.pdf.

Saavedra Martínez MA. 2006. Manejo del Cultivo de Tilapia. Managua, Nicaragua. [consultado 2019 ene 11]. Disponible en: https://www.crc.uri.edu/download/MANEJO-DEL-CULTIVO-DE-TILAPIACIDEA.pdf

Recibido septiembre 6, 2018; aceptado noviembre 6, 2018.

Cómo citar: Paz PE, Martínez Turcios AD, Chávez Chávez JI. 2019. Producción de tilapia (Oreochromis niloticus L.) en la etapa de engorde con dos estrategias de alimentación. Ceiba. 0843:1-6. 